Matthias Harbeck* und Matthias Kaun

\title{
Forschungsdaten und Fachinformationsdienste - eine Bestandsaufnahme
}

https://doi.org/10.1515/bfp-2019-2015

Zusammenfassung: Forschungsdaten sind in all ihrer fachlichen Heterogenität inzwischen bei fast allen Fachinformationsdiensten ein Thema. Schwerpunkte der Auseinandersetzung sind die Kommunikation von Anforderungen, Schwierigkeiten und Möglichkeiten des Forschungsdatenmanagements in die Fächer hinein sowie der Nachweis von Forschungsdaten in fachlichen Rechercheportalen. Aber auch am Aufbau von Infrastrukturen zur Archivierung und Bereitstellung von Forschungsdaten sind Fachinformationsdienste zunehmend beteiligt. Dies belegen die vorgestellten Beispiele und Entwicklungen. Im Hinblick auf eine national organisierte Forschungsdateninfrastruktur verstehen sich die Fachinformationsdienste als geeignete und bereitwillige Kooperationspartner an der Schnittstelle zwischen Fachcommunities und Infrastrukturen. ${ }^{1}$

Schlüsselwörter: Forschungsdaten; Fachinformationsdienst; Nationale Forschungsdateninfrastruktur

\section{Research Data and Specialised Information Services}

Abstract: Research data, in all its disciplinary heterogeneity, is in almost all Specialised Information Services part of the agenda. Key aspects of the discussion include the communication of requirements, difficulties and possibilities of research data management towards academic communities as well as the integration of the research data's metadata into research portals. Furthermore, Specialised Information Services are increasingly involved in infrastructure-building for archiving and accessing research data. The examples presented in this article clearly demonstrate these developments. With regard to a nationally organized research data infrastructure, the Specialised Information Services are suitable and willing cooperation

1 Dieser Artikel wurde mit Unterstützung von Kollegen der AG FID namentlich von Sylvia Daniel, Julia Kreusch, Maxime Mauriège, Ruth Sindt, Sonja Strunk, Stefan Wulle erstellt.

*Kontaktperson: Matthias Harbeck, matthias.harbeck@ub.hu-berlin.de

Matthias Kaun, matthias.kaun@sbb.spk-berlin.de partners ideally positioned with close connections to both academic communities and infrastructures.

Keywords: Research data; specialised information services; German national research data infrastructure; European open science cloud

\section{Einleitung}

Forschungsdaten haben sich in den letzten Jahren fächerübergreifend als ein zentrales Thema wissenschaftlicher Infrastrukturen herauskristallisiert. ${ }^{1}$ Ausgehend von messdatenintensiven Fächern und Forschungsfeldern wie den Natur-, Geo- und Umwelt- aber auch den quantitativ forschenden Sozialwissenschaften hat die Diskussion, wie mit den im Rahmen von Forschungsvorhaben und -projekten erzeugten und bearbeiteten Daten umgegangen werden soll, nun flächendeckend beinahe alle Disziplinen der Wissenschaft erreicht. ${ }^{2}$ Die zunehmende Digitalisierung von Forschung und Lehre erfordert eine aus den jeweils spezifischen Bedürfnissen von wissenschaftlichen Communities heraus entwickelte Forschungsinfrastruktur, in der neue Dienste entwickelt und bestehende effektiv gebündelt werden. Im Zuge der digitalen Transformation des Wissenschaftssystems entstehen und entwickeln sich neue Infrastrukturen, Standards und Modelle für Forschung und Lehre, die wiederum Auswirkungen auf die Wissenschaften selbst und darüber hinaus auf wissenschaftsunterstützende Servicestrukturen haben. ${ }^{3}$ Neben den grundsätzlichen Fragen der Digitalisierung der Wissenschaften und den daraus folgenden Fragen zur Zugänglichkeit von wissenschaftlicher Information, virtuellen Forschungsumgebungen und Open-Access-Publikationen, spielen in allen Wissenschaftsdisziplinen - zunehmend

1 Stäcker und Steenweg (2018).

2 Vgl. dazu die Resonanz der Akteure im deutschen Wissenschaftssystem, u. a. RFII (2018).

3 Die Deutsche Forschungsgemeinschaft (DFG) hat daher explizit eine Expertenkommission und ein internes Projekt zur Erforschung des Digitalen Wandels in den Wissenschaften initiiert, um sich entsprechend neu aufzustellen, vgl. DFG (2018). 
auch in den Geisteswissenschaften - Forschungsdaten eine herausragende Rolle im Wissenschaftssystem.

Die oftmals angeführten Argumente für Überlegungen zum Forschungsdatenmanagement, also dem Umgang mit den anfallenden Forschungsdaten, zielen meist auf vier verschiedene Aspekte ${ }^{4}$ ab:

1. Transparenz der Forschung zum Zwecke der Überprüfbarkeit

2. Transparenz der Forschung zum Zwecke der Nachnutzung

3. Referenzierbarkeit von Forschungsdaten zum Zwecke der guten wissenschaftlichen Praxis

4. Vermeidung von Forschungswiederholungen und damit Entlastung des Steuerzahlers und größere Effizienz

Während deutsche Forschungsfördereinrichtungen diese Entwicklungen zwar wahrnehmen und erste Empfehlungen aussprechen, aber noch keine rigorosen Forderungen nach entsprechenden Datenmanagementplänen und Archivierungslösungen stellen, ${ }^{5}$ sieht dies im Ausland (z. B. der Schweizerischer Nationalfonds SNF) oder auf europäischer Ebene bereits anders aus. ${ }^{6}$

Doch auch in Deutschland haben sich infolge der zunehmenden Beschäftigung mit den Forschungsdaten und der stärkeren Gewichtung der Forschungsdatenarchivierungsfrage verschiedene Akteure bereits Gedanken zu infrastrukturellen Lösungen für die mit einem umfassenden Forschungsdatenmanagement verbundenen, auf die deutsche Forschungslandschaft zukommenden Fragen gemacht. Für verschiedene Disziplinen gibt es bereits fachliche Lösungen, für einige Institutionen oder Länder gibt es erste Ansätze zu institutionellen bzw. Landeslösungen. ${ }^{7}$ Auf nationaler Ebene gibt es im Zuge dieser Entwicklungen Überlegungen für eine Nationale Forschungsdateninfrastruktur (NFDI). Die geplante NFDI (das Förderprogramm hat offiziell am 1.1.2019 begonnen ${ }^{8}$ ) wird kooperativ von verschiedenen Partnern getragen: auf der einen Seite von der Wissenschaft, insbesondere Fachgemeinschaften bzw. Kooperationen zwischen Fachgemeinschaften. Auf der anderen Seite werden Infrastruktur-Partner mit einschlägiger Erfahrung im Umgang mit digitalen (Forschungs-)Daten

4 Vgl. DataFAIRport (o.J.).

5 Vgl. Wissenschaftsrat (2012), 53 oder auch DFG (2015).

6 Vgl. zur Schweizer Förderpolitik und Open Research Data die Angaben des Schweizerischen Nationalfonds (o.J.). Zur europäischen Ebene finden sich Angaben in European Commission (2016).

7 Vgl. die beiden Hefte aus 2018 der Zeitschrift o-bib. Das offene Bibliotheksjournal 5 (2 u. 3).

8 Gemeinsame Wissenschaftskonferenz GWK (2018). identifiziert, die forschungsnahe Services entwickeln und betreiben und in ausgewiesener Weise hervorragend mit der Forschung und der wissenschaftlichen Gemeinschaft verknüpft sind.

Für diejenigen Fächer, für die es noch keine etablierten Datenarchivierungsansätze oder nicht einmal einen entsprechenden fachlichen Diskurs über den Umgang mit Forschungsdaten gibt, haben sich mittlerweile verstärkt die seit 2014 ins Leben gerufenen und von der Deutschen Forschungsgemeinschaft (DFG) geförderten Fachinformationsdienste für die Wissenschaft in die Diskussionen eingebracht. Der oftmals kritisierte oder zumindest in Teilen skeptisch gesehene Umstieg von den Sondersammelgebieten zu den Fachinformationsdiensten (FID), der einen Paradigmenwechsel hin zu einer Projektkultur, die keine Kontinuität gewährleistet und hohen Antragsaufwand erzeugt, birgt gerade für heterogene, an Fachbedarfe angepasste Services im Bereich Forschungsdatenmanagement auch große Chancen. Die zuvor eingeengte Perspektive, unter Informationsversorgung im Wesentlichen die Versorgung mit Printliteratur zu verstehen, wurde nun aufgegeben zugunsten eines breiteren und flexibleren Ansatzes von „Fachinformation“, der auch bibliografische Daten, digitale Angebote und eben Forschungsdaten beinhaltet. Mit dem zudem flexibleren Mitteleinsatz in dem neuen Förderprogramm können nun auch Personal und technische Infrastruktur finanziert werden - beides notwendige Voraussetzungen für die Aufgaben im Bereich „Forschungsdatenmanagement" und neben den Anregungen aus den jeweiligen Fachcommunities Grund dafür, dass dieses Themengebiet in ihre Projekte aufgenommen werden konnte. Aus der äußerst heterogenen Natur der wissenschaftlichen Disziplinen und der in ihnen erzeugten „Daten“9 resultieren höchst unterschiedliche Datenformate und Anforderungen an ihre Archivierung und Bereitstellung für mögliche Nachnutzungsszenarien. Für viele Aspekte des Datenmanagements bieten generische Lösungen, wie sie nur initial an übergeordneten Einrichtungen wie Universitäten aufgebaut werden können, oftmals keine zufriedenstellende und hinreichende Antwort.

FID können in diesem Kontext ganz unterschiedliche Rollen einnehmen - je nachdem, wie weit der Diskurs im jeweiligen Fach fortgeschritten ist, welche Archivierungslösungen bereits zur Verfügung stehen und wie spezifisch die fachlichen Anforderungen an das Forschungsdatenmanagement ausfallen. Durch ihre oftmals jahrzehntelange (in den meisten Fällen bereits im Vorgängersystem der

9 Je nach Disziplin kann die Definition des Datenbegriffes sehr unterschiedlich ausfallen. 
Sondersammelgebiete etablierte) Beziehung zu den Fachcommunities und die häufig bereits seit den frühen 2000ern aufgebauten und in den Fächern verankerten fachlichen Rechercheportale lassen sich viele der kommunikativen Prozesse beim Aufbau einer fachlichen aber auch einer nationalen - Forschungsdateninfrastruktur sowie der Nachweis entsprechender Forschungsdatensätze gut bei den FID ansiedeln oder unter Einbeziehung ihrer Expertise bewältigen.

\section{Herangehensweisen in den Fachinformationsdiensten: Beispiele für Verschiedenartigkeit, unterschiedliche Geschwindigkeiten und gemeinsame Probleme}

Die meisten FID verfolgen und berücksichtigen heute das breite Spektrum um das Thema Forschungsdaten und die dazugehörenden Anforderungen an eine Informationsinfrastruktur aktiv. Manche FID haben bereits in ihrer ersten Förderphase einen Schwerpunkt auf Forschungsdaten gelegt, andere FID behandeln das Thema aktiv, aber nicht immer schwerpunktmäßig. Nahezu alle FID aber verfolgen das Thema und die an Forschungsdaten angrenzenden Themenfelder spätestens mit der jetzt für fast alle FID angebrochenen zweiten Förderphase. Im Folgenden werden beispielhaft Strategien und Erkenntnisse aus der Arbeit einzelner Fachinformationsdienste zum Forschungsdatenmanagement zusammengeführt und vorgestellt, um ein besseres Verständnis für gemeinsame Probleme einerseits und unterschiedliche Herangehensweisen und Lösungsansätze andererseits zu generieren. Basierend auf Perspektiven und Arbeitsständen aus neun verschiedenen FID, die ganz unterschiedliche Aspekte des Themas Forschungsdatenmanagements deutlich machen, sollen die Aktivitäten matrixartig zusammengestellt werden. Bei den neun FID handelt es sich um folgende:

- $\quad$ FID Asien (Berlin und Heidelberg)

- FID Bildungsforschung und Erziehungswissenschaft (Frankfurt/M., Erlangen-Nürnberg, Braunschweig, Berlin)

- FID Geschichte und FID Altertumswissenschaften (München)

- FID Musikwissenschaft (München)

- FID Nordeuropa (Kiel)

\author{
- FID Ost-, Ostmittel- und Südosteuropa (München) \\ - FID Pharmazie (Braunschweig) \\ - FID Philosophie (Köln) \\ - FID Sozial- und Kulturanthropologie (Berlin) \\ - FID Soziologie (Köln)
}

\subsection{Sensibilisierung für und Kommunikation um das Thema Forschungsdaten}

Was z.B. für die Meteorologie seit Jahrzehnten zum Standard gehört, ist gerade für Geisteswissenschaften teilweise noch Neuland: Forschungsdaten, die fachspezifische Definition was Forschungsdaten sind, wie diese behandelt werden sollten, wie Forschungsdaten gesichert, ggf. geschützt, ggf. geteilt, suchbar und auch angeboten werden sollten - dies alles sind Themen, denen man sich in den letzten Jahren und insbesondere seit Beginn der Aktivitäten des Rats für Informationsinfrastrukturen (RfII) verstärkt gewidmet hat. Alle FID haben in den zurückliegenden Jahren mit ihren Fachbeiräten, Wissenschaftlern und Fachverbänden das Thema Forschungsdaten adressiert und somit in Diskussionen dazu beigetragen, dass die Vertrautheit mit dem Thema in den unterschiedlichsten Disziplinen gestiegen ist. Die FID sind bestens mit der Wissenschaft vernetzt und können so ihre Kommunikations- und Aktionskanäle nutzen und Themen, die übergreifend für die Wissenschaft und Informationsinfrastrukturen gleichermaßen relevant sind, aufgreifen. Während z.B. der FID Pharmazie zu einem frühen Zeitpunkt aktiv wurde, um Bedarfe der Fachcommunity im Bereich Forschungsdatenmanagement auszuloten, wurde über den Fachbeirat deutlich, dass ein starkes Interesse an spezifischen Unterstützungsleistungen im Bereich Forschungsdaten besteht. Nachdem der FID Sozial- und Kulturanthropologie das Thema bereits in der ersten Förderphase bis 2018 aktiv verfolgt und in die Wissenschaft kommuniziert hat, wurde der Disziplin dabei bewusst, wie groß das Gefälle unter ihnen in Bezug auf Wissen und Dringlichkeit des Themas ist. Infrastrukturell wurde zudem weitestgehend ethnographische Neuland betreten: Ähnlich anderen Disziplinen gibt bzw. gab es kein etabliertes Fachrepositorium für disziplinäre Forschungsdaten auf nationaler Ebene, trotz durchaus verbreiteter digitaler Arbeitsmethoden. Es fehlt bzw. fehlte das Bewusstsein für die Notwendigkeit einer langfristigen Speicherung und der Möglichkeit der Nachnutzung von Daten. Für die zweite Förderphase wurden daher einerseits die Kommunikationsaufgaben verstetigt und andererseits eine technische Partnerschaft mit dem Datenservicezentrum Qualiservice der Universität Bremen angestrebt (und bewilligt), die fachlich spezifische Archi- 
vierungs- und Bereitstellungslösungen bieten soll. Das verfolgte Ziel des FID Philosophie im ersten Förderzeitraum (2018-2020) ist es, einen qualifizierten Umgangs mit Forschungsdaten im Fach Philosophie zu etablieren und die Entwicklung entsprechender Infrastruktur (u.a. zur Überwindung der bestehenden zersplitterten Forschungsdatenlandschaft aufgrund dezentraler Ansätze und somit zur Vereinheitlichung von Standards und Methoden) anzustoßen.

Andere FID machten ähnliche Erfahrungen und verstehen sich selbst dabei als Partner der Wissenschaft im Umgang mit Forschungsdaten. Aufgrund ihrer jahrelangen Expertise mit Daten, Konvertierungen, Werkzeugen und der Generierung von Angeboten an die Wissenschaft, bringen sich FID aktiv in die Diskussion und Planung der NFDI ein.

\subsection{Vernetzung und Best Practices}

FID stehen, besonders dann, wenn sie schon im Kontext des SSG Programms ähnlich verantwortlich waren, hervorragend mit der Wissenschaft im Austausch. Neben der wichtigen Aufgabe der Informations- und Literaturvermittlung und -versorgung kommen heute vielfältige neue Aufgaben auf die FID zu. Als aktiver Teil der informationstechnischen Infrastruktur und mit ihren engen Kontakten in die Wissenschaft können sie so die Aufgaben der Vernetzung zwischen Informationsinfrastruktur und Wissenschaft besonders gut wahrnehmen und auch Hilfestellungen leisten. Die FID arbeiten als Vermittler zwischen den verschiedenen Akteuren im Kontext Forschungsdaten. Sie

- nehmen eine Vermittlungsfunktion zu Fachgemeinschaften und Multiplikatoren (u. a. zu Fachreferaten in Bibliotheken) ein;

- erheben, wie und in welchen Formaten die Wissenschaft Daten produziert und welche Schwierigkeiten im Umgang mit diesen Daten bestehen (können);

- eruieren, wie diese Daten aus üblichen Forschungstools extrahiert und archiviert werden können, idealerweise mit Blick auf die Disziplin und auch unter rechtlichen Gesichtspunkten;

- kümmern sich um die Einbindung der Nachweise von Forschungsdaten in vorhandene Informations- und Kommunikationsinfrastrukturen und die fachspezifische Kontextualisierung von Forschungsdaten (z.B. Verknüpfung von Forschungsdaten mit Publikationen, Projekten, Instrumenten) unter Berücksichtigung von Open-Science-Merkmalen (Open Access, Open Data u.a.);
- erarbeiten Handlungsempfehlungen oder Best-Practice-Beispiele zum Umgang mit und zur Archivierung von Forschungsdaten für die Forschenden;

- unterstützen bei der überregionalen Lizenzierung von Forschungsdatenbeständen und -instrumenten (bspw. von psychologischen Tests, Fragebogenitems) als Service für die Fachcommunities.

Das geschieht in allen hier berücksichtigten FID im engen Austausch in und mit den Fachbeiräten sowie den Fachverbänden und/oder einzelnen Wissenschaftlern.

\subsection{Technische Lösungsansätze (Metadaten, Repositorien, Langzeitarchivierung, Nachweissysteme)}

Neben der in ihrer Wirkung und Wichtigkeit nicht zu unterschätzenden Kommunikationsaufgaben sind manche FID bereits auch in der Entwicklung von technischen Lösungsansätzen für Forschungsdaten aktiv. Der FID Asien ist derzeit auf zwei Wegen unterwegs: Die UB Heidelberg bietet die dauerhafte Speicherung und Verwaltung von Forschungsdaten sowie deren Verknüpfung zu den zugehörigen Online-Publikationen in Zusammenhang mit ihren verschiedenen OA-Publikationsplattformen als Service an. Die Staatsbibliothek zu Berlin hat darüber hinaus die Speicherstruktur (Integriertes Textrepositorium $\mathrm{ITR}^{10}$ ), in der u.a. mehrere Millionen lizenzierte Objekte verwaltet werden, so angelegt, dass Forschungsdaten im Kontext der gespeicherten und archivierten Volltext- oder Bildtextdaten z.B. in Form von Transkriptionen, Exzerpten, Auswertungen usw. im ITR mit Verknüpfung zu den Quellmaterialen sowie den inhaltlich verbundenen Medien gespeichert werden.

Die UB Braunschweig mit ihrem FID Pharmazie kooperiert für den Aufbau eines elektronischen Laborjournals zur Unterstützung der Fachcommunity im Forschungsdatenmanagement sowohl mit dem KIT Karlsruhe im DFGgeförderten Projekt Chemotion als auch dem Institut für Medizinische und Pharmazeutische Chemie der Universität Greifswald.

Die Bayerische Staatsbibliothek (BSB) wird ab 2019 gemeinsam mit vier außeruniversitären Forschungsinstitutionen ${ }^{11}$ einen Dienst für Forschungsdaten der deutschen

10 CrossAsia ITR (2019).

11 Die Partner sind das Collegium Carolinum, das Leibniz-Institut für Geschichte und Kultur des östlichen Europas, das Herder-Institut für historische Ostmitteleuropaforschung und das Leibniz-Institut für Ost- und Südosteuropaforschung. 
Ost-, Ostmittel- und Südosteuropaforschung aufbauen. Ein entsprechender Förderantrag wurde Ende 2018 von der DFG bewilligt. OstData beinhaltet eine modulare und netzwerkartige technische Infrastruktur zur Speicherung, Veröffentlichung, Langzeitarchivierung sowie zur Suche von Forschungsdaten. Hinzu kommen vielfältige Beratungsleistungen für die entsprechende wissenschaftliche Community. Hier liegt also ein Beispiel für eine neu angestrebte Lösung vor, die aber nicht allein im FID-Kontext, sondern mit weiteren Partnern realisiert wird. Es ist offensichtlich, dass ohne die Ausrichtung des FID und die entsprechende Strategie der Trägereinrichtung Bayerische Staatsbibliothek ein solcher komplementär zum FID-Programm gestellter Antrag nicht denkbar gewesen. Auch die anderen FID an der BSB wie der FID Geschichte und FID Musikwissenschaft profitieren von diesen Rahmenbedingungen, so dass auch diese FID in Gesprächen und Verhandlungen mit der Wissenschaft, den Fachverbänden und weiteren Akteuren sind.

Neben der Bereitstellung zielführender Informationsangebote und der Präsentation bestehender Angebote zur Archivierung und Bereitstellung standardisierter Forschungsdaten plant der FID Soziologie, auf seiner Portalseite SocioHub ein zentrales Recherche- und Nachweissystem bereitzustellen, das über Literaturinformationen hinaus empirische Daten sowie die aktuelle Forschungslandschaft der Soziologie abbildet. Auf diese Weise soll die Nachnutzbarkeit bereits erhobener Forschungsdaten optimiert und die Generierung neuen Wissens ohne z.T. kostenintensive Datenneuerhebungen erleichtert werden.

Der FID Nordeuropa an der UB Kiel erarbeitet derzeit gemeinsam mit dem Hamburger Zentrum für Sprachkorpora (HZSK) an der Universität Hamburg und den vier großen nordeuropäischen Clarinzentren ${ }^{12}$ eine Vorlage für ein Metadatenschema für Sprachressourcen, das dann über das HZSK gemeinsam in Clarin weiterdiskutiert werden soll. Die im Bereich der Sprach- und Literaturwissenschaft in Teilen schon recht weit fortgeschrittene Diskussion zum Forschungsdatenmanagement und die durch Projekte wie Clarin-D und DARIAH-DE vorangetriebene technische Beschäftigung mit Fragen des Umgangs mit digitalen Daten

12 Clarin ist eine europäische Infrastrukturmaßnahme für die Geistes- und Sozialwissenschaften, die mit verschiedenen Digital-Humanities-Projekten vor allem im Bereich der Sprach- und Literaturwissenschaft neue digitale Forschungsmöglichkeiten und -felder eröffnet und etabliert hat. Clarin steht für „Common Language Resources and Technology Infrastructure“, die hierbei entstandenen digitalen Textkorpora, ihre Annotationen und weiteren digitalen Verarbeitungsschritte können in diesem Kontext als Forschungsdaten begriffen werden. verdeutlicht, dass hier schon Detailfragen des Managementprozesses geklärt werden.

Ähnlich anderen bringt der FID Erziehungswissenschaft und Bildungsforschung sich in dem Verbund Forschungsdaten Bildung (VerbundFDB) ein, an dem das Leibniz-Institut für Bildungsforschung und Bildungsinformation (DIPF) mit dem Forschungsdatenzentrum Bildung (FDZ) beteiligt ist und die Gesamtkoordination des VerbundFDB verantwortet. Mit Blick auf die zukünftige NFDI und mit Rückgriff auf die bereits erfolgreiche Implementierung von vernetzten Datendiensten in der Bildungsforschung, strebt der Rat für Sozial- und Wirtschaftsdaten (RatSWD) in dem interdisziplinären Kontext der Sozial-, Wirtschafts- und Verhaltenswissenschaften eine erweiterte föderierte Archivierungsinfrastruktur an. Der FID Erziehungswissenschaft und Bildungsforschung visiert an, diese bereits eingeleitete Weiterentwicklung für den gesamten Disziplinenraum zu unterstützen.

\section{Die Fachinformationsdienste und die Nationale Forschungsdateninfrastruktur}

Die neu entstehende NFDI wird die Wissenschaftslandschaft nachhaltig verändern und tief prägen. Eine Mitwirkung in dieser Infrastruktur würde die jeweilige FID-Bibliothek langfristig in einem zentralen Bereich der Informationsinfrastruktur verankern und böte erhebliche Entwicklungschancen. Wie die Beispiele zeigen, haben fast alle FID die Entwicklungen der NFDI bei ihrem Engagement im Forschungsdatenmanagement bereits im Blick und kommunizieren diese Entwicklungen in ihre Fachgemeinschaften hinein, ganz egal wie weit fortgeschritten ihre Bemühungen oder auch die fachlichen Diskurse bereits sind.

Fachinformationsdienste bieten als wissenschaftliche Infrastruktureinrichtungen für Spezialliteratur und forschungsrelevante Informationen mit fachlicher und nationaler Ausrichtung einen zentralen Baustein für eine zukünftige NFDI und können eine passende Ergänzung der maßgeblich auf Forschungsdatenservices ausgerichteten Vorhaben sein. Über ihre Bedarfsorientierung und ihren engen Austausch mit den Fachcommunities hinaus verfügen die beteiligten wissenschaftlichen Bibliotheken und Infrastruktureinrichtungen, die im Wesentlichen die FID tragen, bereits heute über eine anerkannt leistungsstarke, polyzentrische Informationsinfrastruktur und sind prädestiniert, um mit ihren vielfältigen, auf die jeweiligen wissenschaftlichen Communities zugeschnittenen Services ei- 
ne exponierte Rolle bei der Erarbeitung und Bereitstellung disziplinspezifischer Lösungen auch zum Umgang mit Forschungsdaten sowie deren Verarbeitung und langfristiger Sicherung einzunehmen. Wie oben gezeigt, bieten manche FID bereits Forschungsdatenservices an. Über die FID besteht daher ein konkretes Potenzial, spezifische fachliche Schnittstellen zwischen bestehenden Forschungsdateninfrastrukturen und Fachgemeinschaften zu bilden.

Bei allen Entwicklungsstadien des Aufbaus einer NFDI, die sowohl als strategischer, aber auch als kooperativ-planerischer Prozess gesehen wird, können die FID als aktiver Partner zusammen mit der Wissenschaft und den Infrastrukturen innerhalb der sich formierenden NFDI-Konsortien agieren - obwohl ihre Struktur unter den gegebenen Förderbedingungen auf geringe (Projekt-)Lebenszyklen angelegt ist. Nichtsdestotrotz verspräche die an den FIDBibliotheken bereits existierende Fachexpertise sowie die bereits aufgebauten Infrastrukturen (z. B. die Portale) ein gutes „return on investment“, wenn FID-Bibliotheken im Rahmen einer - im besten Fall langfristigen - Förderung in den NFDI-Strukturen mitwirkten.

Die Arbeitsgemeinschaft der Fachinformationsdienste (AG FID) hat die FID in ihrem Positionspapier vom September $2018^{13}$ mit ihrer fach- bzw. regionalspezifischen Ausrichtung und Expertise als kooperativ Handelnde und Mitwirkende im NFDI-Netzwerk hervorgehoben. Als Handlungsfelder im Prozess der Planung, des Aufbaus und des Betriebs der NFDI sehen die Mitglieder insbesondere folgende, schwerpunktmäßig informationswissenschaftliche und bibliothekarische Arbeitsschritte, die sich auch in den oben angeführten Einzelbeispielen wiederfinden:

- Aufbau, Betrieb und Weiterentwicklung fach- bzw. regionalspezifischer Dienste und Infrastrukturen wie z.B. Repositorien, Open-Access-Publikationsplattformen, Nutzbarmachung lizenzpflichtiger Daten, spezifische Suchsysteme (Discovery Services), Virtuelle Forschungsumgebungen oder Kommunikationsinfrastrukturen in enger Abstimmung mit der Wissenschaft

- Lizenzierung von Datenbanken und E-Ressourcen und ggf. die Überführung dieser lizenzierten Daten in Forschungsdatenarchive zur weiteren Nachnutzung (wie z. B. NatHosting)

- Datenmanagement inkl. der Analyse, Kontextualisierung, Transformation und dem Prozessieren von Metadaten sowie anderen Daten

13 Arbeitsgemeinschaft der Fachinformationsdienste der Sektion 4 des DBV (2018).
- Erschließung von Daten und Datendistribution in andere Kontexte und Angebote (Interoperabilität), Vergabe von persistenten Identifikatoren

- Entwicklung von Workflows, Standards inkl. Metadatenstandards, Werkzeugen

- Dialogpartner für die Wissenschaften und die Partner im NFDI-Netzwerk zu Anforderungen der Wissenschaft im Kontext Daten- und Rechtemanagement

- Dialogische Beratung über und aktive Unterstützung bei diesen Services, insbesondere zu Erschließung und Werkzeugen

- Durchführung von Bedarfserhebungen, Workshops, Expertengesprächen mit den Zielgruppen

- Koordination der Kommunikation mit wissenschaftlichen Communities

Die in der AG FID organisierten Fachinformationsdienste sehen sich als integrale Bestandteile einer ebenfalls polyzentrisch konzeptionierten NFDI und sind zum Teil bereits in mehr oder weniger konkrete Konsortialbildungen, ${ }^{14}$ aber auf jeden Fall im Themenkomplex Forschungsdaten involviert. Aufgabe der FID wird über die o.g. Punkte hinaus also zweierlei sein: Zum einen die im Prozess der Planung und des Aufbaus der NFDI entwickelten Lösungen in ihre jeweilige Zielgruppe rückzukoppeln und als Multiplikatoren in die Fachcommunity hineinzuwirken und zum anderen dafür zu sorgen, dass die spezifischen Anforderungen an derartige Infrastrukturen, die durch die multidisziplinäre, regionale und theoretische Heterogenität der von den FID versorgten Fächer bedingt ist, berücksichtigt werden. Diese Entwicklung ist als dynamischer Erfahrungs- und Entwicklungsprozess zu verstehen, der zumindest zu Beginn - noch keinen allzu starren Auflagen unterliegt, aber bereits Standardisierungsmöglichkeiten im Blick haben sollte (z. B. Metadatenschemata und Austauschformate für die Integration in Nachweissysteme zur besseren Sichtbarkeit).

Um diese Aufgaben verlässlich als Partner im Wissenschaftssystem zu erledigen, bedarf es finanzieller, organisatorischer, personeller und technischer Sicherheiten, damit u. a. auch die FID dauerhaft ihren Beitrag beim Aufbau einer kooperativ organisierten Speicherstruktur und seiner Vermittlung in die Forschungslandschaft leisten können. Die vielfältige Expertise der FID im Bereich des Forschungsdatenmanagements bzw. im Umgang mit Forschungsdaten, die sich auch in den skizzierten Beispielen

14 Um zwei Beispiele zu nennen, der FID Pharmazie steht im engen Austausch mit dem Konsortium NFDI4Chem und der FID Musikwissenschaften mit NFDI4Culture. 
aus den einzelnen Einrichtungen spiegelt, demonstriert deutlich, dass die FID wertvolle Partner im Kooperationsnetzwerk zum Aufbau der NFDI sein wollen und können. Die Flexibilität des seit 2014 laufenden FID-Förderprogrammes der DFG hat dies ermöglicht. Um diese Rolle abzusichern, sehen die FID die Notwendigkeit zur zügigen Weiterentwicklung der existierenden Förderprogramme hinsichtlich einer verlässlichen Verstetigung bei gleichzeitiger Beibehaltung flexibler Förderelemente, um Innovation und Heterogenität gerecht zu werden. Nur dann können die FID systematische Verbindung zwischen Fachdisziplinen und institutionalisierter Informationsinfrastruktur in Deutschland darstellen und dabei mitwirken, dass Forschungsdaten verantwortungsbewusst und verlässlich Bestandteil der deutschen Forschungslandschaft in ihrer fachlichen Gesamtheit werden können.

\section{Literaturverzeichnis}

Arbeitsgemeinschaft der Fachinformationsdienste der Sektion 4 des DBV (2018): Gemeinsame Stellungnahme der FID leitenden Einrichtungen und der AG Fachinformationsdienste (AG FID) zur Einbindung von Fachinformationsdiensten in den Aufbau einer Nationalen Forschungsdateninfrastruktur. Verfügbar unter http s://wikis.sub.uni-hamburg.de/webis/images/a/a1/AG_FID_zu_ NFDI.pdf.

Bambey, Doris; Corti, Louise; Diepenbroek, Michael; Dunkel, Wolfgang; Hanekop, Heidemarie; Hollstein, Betina; Imeri, Sabine; Knoblauch, Hubert; Kretzer, Susanne; Meier zu Verl, Christian; Meyer, Christian; Meyermann, Alexia; Porzelt, Maike; Rittberger, Marc; Strübing, Jörg; Unger, Hella von; Wilke, René (2018): Archivierung und Zugang zu Qualitativen Daten.

CrossAsia ITR (2019): Verfügbar unter https://blog.crossasia.org/ka tegorie/itr-und-entwicklungen.

DataFAIRport (o.J.): Verfügbar unter http://www.datafairport.org.

DFG (2015): Leitlinien zum Umgang mit Forschungsdaten. Verfügbar unter http://www.dfg.de/download/pdf/foerderung/antragstel lung/forschungsdaten/richtlinien_forschungsdaten.pdf.

DFG (2018): DFG setzt Expertenkommission „Wissenschaft im digitalen Zeitalter" ein: Differenzierte Analyse des digitalen Wandels in den Wissenschaften/Auftaktsitzung in Bonn. Pressemitteilung Nr. 48.

European Commission (2016): Horizon 2020: Aktualisiertes Arbeitsprogramm unterstützt Wettbewerbsfähigkeit durch offene Wissenschaft. Verfügbar unter http://europa.eu/rapid/press-rel ease_MEMO-16-2604_de.htm.
Gemeinsame Wissenschaftskonferenz GWK (2018): Forschungsdaten nachhaltig sichern und nutzbar machen - Startschuss für eine Nationale Forschungsdateninfrastruktur. PM 13/2018. Berlin, Bonn.

Harbeck, Matthias; Imeri, Sabine; Sterzer, Wjatscheslaw (2018): Feldnotizen und Videomitschnitte. Zum Forschungsdatenmanagement qualitativer Daten am Beispiel der ethnologischen Fächer. In: o-bib. Das offene Bibliotheksjournal, 5 (2), 12341. doi: 10.5282/O-BIB/2018H2S123-141.

RFII (2018): Rat für Informations-Infrastrukturen: Themen, Verfügbar unter http://www.rfii.de/de/themen.

Schweizerischer Nationalfonds (o.J.): Open Research Data. Verfügbar unter http://www.snf.ch/de/derSnf/forschungspolitische_posi tionen/open_research_data/Seiten/default.aspx\#Grundsatzerk l\%E4rung\%20des\%20SNF\%20zu\%200pen\%20Research\%20D ata.

Stäcker, Thomas; Steenweg, Helge (2018): Forschungsdaten Aufgabe und Herausforderung für Bibliotheken. In: o-bib. Das offene Bibliotheksjournal, 5 (2), IV-V. doi: 10.5282/O-BIB/ 2018H2SIV-V.

Wissenschaftsrat (2012): Empfehlungen zur Weiterentwicklung der wissenschaftlichen Informationsinfrastrukturen in Deutschland bis 2020 (Drs. 2359-12). Verfügbar unter http://www.wissen schaftsrat.de/download/archiv/2359-12.pdf.

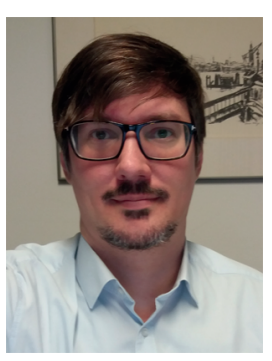

Matthias Harbeck

Universitätsbibliothek der HumboldtUniversität zu Berlin Unter den Linden 6 D-10099 Berlin matthias.harbeck@ub.hu-berlin.de

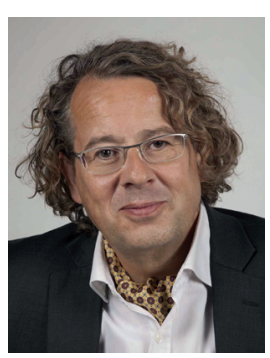

Matthias Kaun

Staatsbibliothek zu Berlin - Preußischer Kulturbesitz Potsdamer Straße 33 D-10785 Berlin matthias.kaun@sbb.spk-berlin.de 